\title{
Physiological sex differences in microglia and their relevance in neurological disorders
}

\author{
Natalia Yanguas-Casás \\ Grupo de Investigación en Linfomas, Instituto Investigación Sanitaria Puerta de Hierro-Segovia de Arana (IDIPHISA), Majadahonda \\ 28222, Spain.
}

Correspondence to: Dr. Natalia Yanguas-Casás, Grupo de Investigación en Linfomas, Instituto Investigación Sanitaria Puerta de Hierro-Segovia de Arana (IDIPHISA), Majadahonda 28222, Spain.E-mail: nyanguas@idiphim.org

How to cite this article: Yanguas-Casás N. Physiological sex differences in microglia and their relevance in neurological disorders. Neuroimmuno/ Neuroinflammation 2020;7:13-22. http://dx.doi.org/10.20517/2347-8659.2019.31

Received: 31 Dec 2019 First Decision: 5 Feb 2020 Revised: 11 Feb 2020 Accepted: 17 Feb 2020 Published: 21 Mar 2020

Science Editor: Jeffrey Bajramovic Copy Editor: Jing-Wen Zhang Production Editor: Tian Zhang

\begin{abstract}
Microglia are the resident immune cells in the brain and maintain homeostasis and functionality of this tissue. These cells are key producers of immune mediators, such as cytokines and chemokines, are critical for normal brain development, and affect neurogenesis, axonal migration, synapse formation and function, and programmed cell death, among others. Sex differences exist in many of these processes throughout brain development up to adulthood and the aged brain. In the last few years, sex differences in microglia responses, brain colonization, and number and morphology within the developing brain have drawn the attention of researchers as a potential explanation to the sex differences in the brain and due to their potential relevance in the incidence, prevalence, and outcome of many neurological disorders. In this review, we summarize the sex differences of microglial cell functions and their potential relevance in physiological as well as pathological conditions in the brain.
\end{abstract}

Keywords: Microglia, sex differences, functional responses, neurological disorders

\section{INTRODUCTION}

There is a differential sex-susceptibility, penetrance, and outcome in neurological disorders. An important inflammatory component goes along with these disorders, and microglia, the immune resident cells of the Central Nervous System (CNS), play a pivotal role in the triggering and resolution of neuroinflammatory processes $^{[1]}$. As the main regulators of immune responses in the CNS, they have come into focus recently due to their potential contribution to the sex differences found in neurological disorders. In this review,

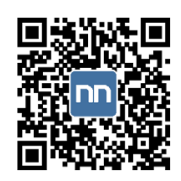


we aim to summarize the sex differences in functional responses of microglia described thus far and their relevance in pathology.

\section{MICROGLIA}

Microglia represent $10 \%-15 \%$ of the cells in the brain ${ }^{[2]}$. In recent years, microglia functions have extensively widened, ranging from mere local immune defense of the CNS to being key players in brain development and physiology. They have been shown to regulate dynamic surveillance of the environment through their active processes, maintaining homeostasis and modulating neuroinflammation ${ }^{[3-6]}$. They also mediate phagocytosis and clearance of debris and apoptotic cells in disease and neurogenic niches ${ }^{[5,7-10]}$, shape brain development through synapse pruning, and allow brain wiring of neuronal circuits ${ }^{[1-13]}$.

Unlike most cell types in the CNS, microglia proceed from myeloid precursors that migrate from the yolk sack to the CNS in early embryological stages [Embryonic Day $8.5\left(\right.$ E8.5) ${ }^{[2,14]}$, before the closure of the blood-brain barrier, which occurs around E13-E14.5 in mice ${ }^{[14,15]}$. Interestingly, microglial brain colonization during embryonic development is highly conserved across vertebrate species ${ }^{[16-19]}$.

Microglial lineage differs from that of macrophages, as it is driven by the cytokine macrophage colony stimulating factor (M-CSF), as well as the transcription factors Pu.1, Irf8, and Sall $1^{[14,20,21]}$. Microglia are self-renewed from local proliferation of CNS resident cells, and the turnover is relatively low in both humans and rodents. This suggests that these cells are likely to be primed, or even have a memory, due to the different events they are exposed to through their lifespan ${ }^{[22-24]}$. This microglia priming will be determinant in their responses, both in physiological conditions and in disease ${ }^{[25]}$.

Microglia represent most of the fetal glial population, especially in early developmental stages ${ }^{[26]}$. Microglia roles at this time are likely to be sex-specific as sex differences may arise as early as hematopoiesis in the embryonic yolk sac or when CNS colonization occurs during early embryonic development ${ }^{[26-28}$. Moreover, male and female microglia follow temporarily different trajectories during development ${ }^{[29,30]}$. Microglia influence sexual differentiation; indeed, masculinization of the brain is dependent on the activation stage of these cells ${ }^{[28,31-34]}$.

Fetal gonads develop early in development, and in males are fully active (except for spermatogenesis) by mid to late gestation, showing a surge in fetal testis androgen production beginning the last few days of gestation and enduring until shortly after birth in rodents. In primates, androgen production occurs from the end of the first trimester and well into the second with another peak at $\operatorname{birth}^{[35,36]}$. Once in the brain, testosterone $(\mathrm{T})$ can be either aromatized to estradiol (E2) or 5- $\alpha$ reduced to dihyrdotestosterone (DHT). Both T and DHT induce some masculine endpoints but it is E2 that is the dominant masculinizing hormone in the rodent brain, through a prostaglandin E2 (PGE2)-mediated process ${ }^{[37,38]}$. Morphologically, in certain sex differentiated brain regions, such as the preoptic area, males have more microglia with an "activated" morphology characterized by an increase in cell body size and a decrease in process length and branching $^{[33]}$.

Recent studies have demonstrated that microglia density and phenotype vary between male and female rodents in several brain areas ${ }^{[33,39,40]}$. Mid adolescent changes lead to a higher blood flow in women compared to men, which is maintained throughout life until the 60s, when this difference is milder. These differences in blood flow may play a role in differential microglia density in certain brain areas ${ }^{[41-43]}$. Despite this, not much attention has been paid to the relevance of sex differences in blood flow or vasculature in differential microglia infiltration during fetal development. Subtle changes in the timing and density of microglia arrival to certain brain regions, as a result of differential blood flow, would lead to differential interaction of these cells with progenitors at different stages of microglia or neural progenitor 
Table 1. Sex differences in the incidence of neurological disorders in humans

\begin{tabular}{ll}
\hline Male brain & \multicolumn{1}{c}{ Female brain } \\
\hline Autism Spectrum Disorders (4:1) & Alzheimer's Disease (3:1) \\
Parkinson's Disease (3:1) & Depression (2:1) \\
Attention Deficit Hyperactivity Disorder & Anxiety (2:1) \\
Attention Deficit Hyperactivity Disorder (3:1) & Multiple Sclerosis (2-3:1) \\
Schizophrenia (1.4:1) & \\
Amyotrophic Lateral Sclerosis (1.6:1) & Adult-onset neurological disorders \\
Early-onset neurological disorders & \\
\hline
\end{tabular}

Most frequent neurological disorders in humans. Global prevalence of each disorder is shown in parenthesis as the ratio of men vs women (left side) or women $v s$. men (right side)

differentiation, resulting in sex specific microglia subpopulations in different brain areas. This is relevant because microglia phenotypes vary across regions of the CNS, in disease as well as in physiological conditions at different stages in life, especially in early development and aging, which are two critical life stages for the appearance of neurological disorders, in both humans and rodents ${ }^{[44-47]}$.

\section{Sex differences in neurological disorders}

There is an increasing concern for the real relevance of experimental results obtained in current research. Experimental procedures are often done using only one sex, and results are often extrapolated to both sexes without solid grounds. Several funding agencies, such as the European Commission, the Canadian Institutes of Health Research, and the US National Institutes of Health, have tried to influence researchers to integrate sex/gender not only in clinical research, but also in basic and preclinical research, especially since they identified a sex bias in most clinical trials, usually done in male subjects, in which females are under-represented, leading to mistreatment of women ${ }^{[48,49]}$. In the specific case of neurological disorders, there is a well described sex bias in the prevalence, severity, progression, and outcome of these diseases $[\text { Table } 1]^{[29]}$. Therefore, there is a need of development, implementation, and prioritization of treatments and preventive interventions specific for sex, age, and population to reduce the burden from these disorders $^{[50]}$.

Many early-onset neurodevelopmental disorders show a strong sex-bias toward males ${ }^{[51]}$ while adultonset neurological disorders are female biased ${ }^{[52]}$. As microglia play an important role in both sexual differentiation of the brain and progression of most neurological disorders ${ }^{[27,33,53,54]}$, it is critical to understand how the dynamics and potential dysfunction of microglia at certain developmental points affect the onset and progression of these disorders.

Women have a higher prevalence of Alzheimer's disease (AD, 1.6-3:1 ratio compared to men $)^{[55,56]}$, autoimmune diseases such as multiple sclerosis (MS, 2-3:1 ratio) ${ }^{[57]}$, or mood related disorders such as depression or anxiety disorders $(2: 1)^{[58,59]}$. On the other hand, men are more prone to suffer from Parkinson's disease (PD, 3.5:1 compared to women ${ }^{[60,61]}$, motor neuron disorders such as amyotrophic lateral sclerosis (ALS, 1.6:1 ${ }^{[6,63]}$, autism spectrum disorders (ASD, 4:1 ${ }^{[64-66]}$, attention deficit hyperactivity disorder $(3: 1)^{[67-70]}$, or schizophrenia $(1.4: 1)^{[71,72]}$.

Beyond the prevalence of these disorders, women show greater cognitive decline than men with $\mathrm{AD}^{[73]}$ and a slower rate of decline when suffering from $\mathrm{PD}^{[60,61]}$. In this line, women show increased severity of depression or anxiety disorder symptoms, and men show earlier onset of schizophrenia and more severe symptoms along with worse response to antipsychotic drugs than women ${ }^{[8,59,71,74]}$. On the other hand, men suffering from MS have a faster progression of the disease than women ${ }^{[57,75]}$, and women suffering from ALS have worse survival rates than men ${ }^{[62,63]}$. 


\section{Microglia and sexual differentiation of the brain}

Sexual differentiation of the brain is orchestrated by sex chromosomes, gonadal hormones, and early postnatal environment. X chromosome contains the largest number of immune-related genes in the human genome, including Toll-like receptor pathways (BTK, IRAK1, and $I K K \gamma)$ and microRNAs involved in immune regulation ${ }^{[76-78]}$.

$\mathrm{X}$ chromosome inactivation to match gene expression levels between males and females is not random, as previously thought. Indeed, it is the paternal X chromosome that is consistently inactivated in neonatal brains $^{[79]}$. Fifteen percent of the genes in the $\mathrm{X}$ chromosome, particularly immune-related genes such as toll-like receptor 7 (Tlr7), escape inactivation in females ${ }^{[80,81]}$. TLR7 is implicated in miRNA-mediated increased TNF $\alpha$ release, and different expression of Tlr 7 in females may contribute to intrinsic differences in immune response ${ }^{[82]}$. Therefore, male and female microglia are differentially influenced by these factors since early developmental stages ${ }^{[83]}$.

Sex hormones are likely key players in microglia sexual differentiation, independently of their genetic background. Microglia physiologically express steroid hormone receptors, and are therefore sensitive to the effects of both estrogens and testosterone ${ }^{[84]}$. Indeed, hormones are necessary to establish initial sex differences in microglia. Studies by Villa et al. ${ }^{[30]}$ showed that masculinization of female brain at E2 in mice resulted in transcriptionally male microglia in adulthood in those females. Indeed, once differentiated, microglia retain their sex-specific transcriptional profiles even after transplantation into the brain of the opposite sex in adulthood $^{[30]}$.

Interestingly, young adult female microglia maintain their sex differences in the absence of hormones, as their transcriptome is not drastically affected after ovariectomy ${ }^{[30]}$. However, hormone depletion in aged female mice (over 13 months old) induces profound transcriptome changes in these cells, with increased inflammatory phenotypes ${ }^{[85,86]}$. Further studies are required to determine if changes in circulating hormones during aging are responsible for these differences. It would be especially relevant to determine the relevance of this in the incidence of neurodegenerative disorders in women, as these often appear in the postmenopausal period.

In addition, early pre- and postnatal environment is key in the sexual differentiation of the brain. Development at this point involves rapid myelination of neuronal fibers and synaptogenesis, arborization, and pruning. This time of extensive growth is also a critical period where environmental factors, such as nutritional factors (folate and palmitic acid), early postnatal stress, or smoking, can influence optimal CNS development ${ }^{[87,88]}$. Indeed, some functional sex differences in early postnatal microglia are lost upon exposure to palmitic acid $^{[89]}$.

\section{Basal sex differences in microglia functional responses}

There are well described sex differences in microglia in the male and female brain. These differences range from cell density and morphology to different transcription profiles and functions. Transcriptomic data have shown that microglia transcriptome during brain development is characterized by temporal maturation steps that follow different trajectories in males and females: male microglia are developmentally delayed compared with female microglia, starting from E1 ${ }^{[90,91]}$. Besides, the maturation process has features that resemble the pro-inflammatory activation programs typical of adult cells. This is of special relevance, as it suggests a higher sensitivity to inflammatory events in male microglia, which could lead to a faster aging of these cells and affect the risk of disorders ${ }^{[91]}$ [Figure 1].

Microglia density varies significantly across different subregions in the brain in a spatiotemporal fashion. In early developmental stages, microglia density in specific brain areas such as the hippocampus is higher in 
A

MALE MICROGLIA

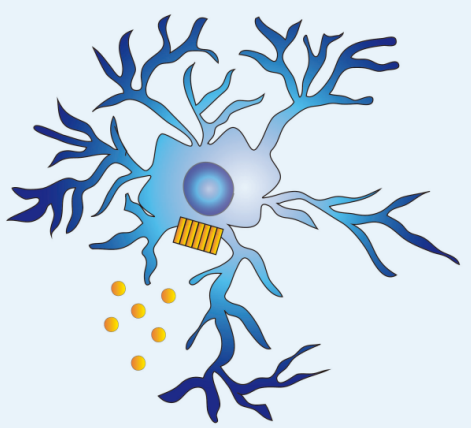

Higher Migration Capacity

More Reactiveness

Enlarged soma

Higher $\mathrm{MHCl}, \mathrm{MHCll}, \mathrm{P} 2 \mathrm{Y} 12$ expression
B
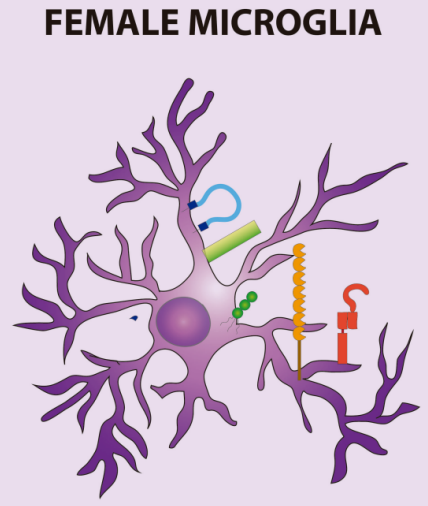

Higher Phagocytic Capacity

Higher expression of phagocytosis receptors

Higher expression of cellular repair and

inflammatory control genes

Figure 1. Physiological sex differences in male and female microglia. A: male microglia have an enlarged soma and more reactiveness in physiological conditions than female microglia. These cells have more pro-inflammatory responses, higher migration capacity, and enhanced $\mathrm{MHCl}, \mathrm{MHCll}$, and $\mathrm{P} 2 \mathrm{Y} 12$ constitutive expression; B: female microglia, on the other hand, have a higher phagocytic capacity and higher gene expression of cell repair and inflammatory control genes

female brains, whereas it is higher in the amygdala in male brains. Other areas, such as the cortex, striatum, and cerebellum, show similar densities in both sexes at these stages. At later developmental stages (early adulthood), there is a higher microglia density in the cortex, hippocampus, and amygdala of male brains, while there are no differences in the striatum and cerebellum ${ }^{[39,92]}$.

Similar to cell density, significant regional heterogeneity has also been found in microglial structural complexity. During development, the soma size of female microglia is larger in the cortex, hippocampus, and amygdala, while there were no changes in microglia size in other areas ${ }^{[39,92]}$. However, in adult stages, male microglia show enlarged somas throughout the brain ${ }^{[39]}$.

Microglial phagocytic activity has been shown to be enhanced in early postnatal female microglia compared with males in both mice and rats ${ }^{[89,93]}$. On the other hand, male microglia show a higher P2Y12 receptor expression and higher motility capacity at this time ${ }^{[89,94]}$. Interestingly, male microglia also show higher MHCI and MHCII expression, as well as enhanced $\mathrm{P} 2 \mathrm{X}$ receptor-mediated signaling, which are indicative of more reactiveness than female microglia already under physiological conditions ${ }^{[39]}$ [Figure 1].

Functional sex differences in microglia may have important functional consequences for disease progression. It is likely that each sex uses different mechanisms to achieve similar baseline functional states adapted to their sex-specific environments, and therefore microglial cells would have equivalent cellular functionality regardless the $\operatorname{sex}^{[95]}$.

Recent work has shown that microglia contribute to sex differences in social behavior ${ }^{[83]}$ and further research will determine to what extent microglia partake in the brain sexual dimorphism. How such intrinsic differences contribute to disease susceptibility also remains to be elucidated ${ }^{[30,39]}$.

\section{Sex differences in microglia responses in disease}

Microglia dysfunction is implicated in every single brain disease. Unveiling microglia functional sex differences in non-physiological states may explain differences in disease susceptibility that result from sex- 
specific inefficient responses. Sexual differentiation of the brain during early development likely underlies the strong sex biases prevalent in many neurological conditions, as they acquire their sex specific identity early in development, which persists during the injury response ${ }^{[30]}$. Therefore, studying sex differences in this context could shed some light on sex-specific disease mechanisms.

Beyond the neuroprotective effect of estrogens per se ${ }^{[96,97]}$, RNA-seq analysis revealed that female microglia express more genes involved in cellular repair and inflammatory control than male microglia, which likely contributes to a more favorable outcome in several injuries ${ }^{[30]}$. Besides, recent studies have shown that male microglia seem to be more reactive already under physiological conditions as well as have a shorter lifespan ${ }^{[39]}$. For example, female microglia show a higher mRNA expression of Shank 3, Fxyd1, Aqp1, or Timp3 and a decreased mRNA expression of Akt1s1, Trem1, S100a9, or Cxcl2, as well as decreased NF- $\mathrm{B}$ activity levels, compared to male microglia ${ }^{[30]}$.

Sex differences in microglia immunomodulatory response to lipopolysaccharide (LPS), a potent proinflammatory agent, have been studied both in vitro and in vivo, and in both conditions male microglia display a higher immune response of male microglia after LPS stimulation ${ }^{[90,98]}$, which is accompanied by greater IL-1 $\beta$ mRNA and MHCI/II expression in male microglia, and decreased CD14 mRNA expression in female microglia ${ }^{[39,98]}$.

Mouse models of forebrain or focal ischemia have shown that young adult female mice and rats sustain lesser injury than males ${ }^{[99-101]}$. Moreover, female microglia display a neuroprotective phenotype in ischemic stroke; indeed, when transplanted in male brains, they protect them from this disease ${ }^{[30]}$.

Something important to keep in mind is that different subsets of microglia respond to various insults such as aging or immune challenges differently. Microglia can be classified into gene expression clusters through the lifespan of the individual. For example, on Postnatal Days 4 and 5, female microglia are enriched for the genes $\mathrm{Cd} 74$, chemokine (C-C motif) ligand $24(\mathrm{Ccl} 24)$, and $\mathrm{Arg} 1^{[102]}$. Interestingly, as the brain ages, there is a progressive expansion of clusters that typically have few very cells in adolescent and adult samples, which are enriched in inflammatory genes and are more responsive to interferon ${ }^{[102]}$. Combination of deep single-cell transcriptome analysis, fate mapping, clonal analysis, in vivo imaging, and transgenic mouse lines have allowed the identification of microglia subsets in different CNS compartments during neuroinflammation ${ }^{[102-104]}$.

Single-cell sequencing of microglia in an Alzheimer's disease mouse model revealed a unique AD-related microglial phenotype, generated by a two-step process involving triggering receptor expressed on myeloid cells 2 (Trem2). Activation is initiated in a TREM2-independent manner involving downregulation of microglia checkpoints, followed by activation of a TREM2-dependent manner ${ }^{[104]}$. The relevance of sex in these unique microglial subsets such as disease-associated microglia remains to be elucidated.

Interestingly, some genes have been linked with sex-specific phenotypes in the case of $\mathrm{AD}$. One such gene is Apoe, which codes apolipoprotein E (ApoE), a modulator of the CNS immune system that can have differential outcomes on microglial function depending on the variant ${ }^{[105-107]}$. The $\varepsilon 4$ variant of the gene, which is expressed more strongly in females, has been linked with a higher risk of developing late-onset $\mathrm{AD}$ in humans ${ }^{[56,108,109]}$. Microglia are a major source of plaque-associated ApoE, which is modulated by TREM2 in AD mouse models ${ }^{[110]}$.

A sex-specific differential expression of Apoe in disease associated microglia has been found in a mouse model of ALS ${ }^{[104,111,112]}$. Microglia isolated from female aged mice also have upregulation of ApoE transcripts compared to males ${ }^{[113]}$. Overall, these findings suggest that $A p o E$ is a gene that could partially explain the sex differences found in $\mathrm{AD}$ and maybe other neurodegenerative disorders. 


\section{CONCLUSION}

Hormonal and genetic environments determine microglia fate to be sex-specific. There are several sex differences in microglia physiology, distribution throughout the brain, functional responses, transcriptional profiles, and sex chromosome composition. Some of these are maintained throughout the lifespan of the individual; however, most of them are dynamic and vary over time. As microglia play a key role in every neurological disease, it is likely that the differences they present contribute to sex differences in the course and incidence of these disorders. Therefore, sex differences in microglia are a new and promising research field to explain the differences in neurological disorders in humans and potentially lead to sex-specific strategies to treat these patients.

\section{DECLARATIONS}

\section{Authors' contributions}

The author contributed solely to the article.

\section{Availability of data and materials}

Not applicable.

\section{Financial support and sponsorship}

None.

\section{Conflicts of interest}

The author declared that there are no conflicts of interest.

\section{Ethical approval and consent to participate}

Not applicable.

\section{Consent for publication}

Not applicable.

\section{Copyright}

(c) The Author(s) 2020.

\section{REFERENCES}

1. Wolf SA, Boddeke HWGM, Kettenmann H. Microglia in physiology and disease. Annu Rev Physiol 2017;79:619-43.

2. Kettenmann H, Hanisch UK, Noda M, Verkhratsky A. Physiology of microglia. Physiol Rev 2011;91:461-553.

3. Davalos D, Grutzendler J, Yang G, Kim JV, Zuo Y, et al. ATP mediates rapid microglial response to local brain injury in vivo. Nat Neurosci 2005;8:752-8.

4. Nimmerjahn A, Kirchhoff F, Helmchen F. Resting microglial cells are highly dynamic surveillants of brain parenchyma in vivo. Science 2005;308:1314-8.

5. Zhao Y, Wu X, Li X, Jiang LL, Gui X, et al. TREM2 Is a Receptor for beta-amyloid that mediates microglial function. Neuron 2018;97:1023-31.e7.

6. Colonna M, Butovsky O. Microglia function in the central nervous system during health and neurodegeneration. Annu Rev Immunol 2017;35:441-68.

7. Asai H, Ikezu S, Tsunoda S, Medalla M, Luebke J, et al. Depletion of microglia and inhibition of exosome synthesis halt tau propagation. Nat Neurosci 2015;18:1584-93.

8. Bolós M, Perea JR, Terreros-Roncal J, Pallas-Bazarra N, Jurado-Arjona J, et al. Absence of microglial CX3CR1 impairs the synaptic integration of adult-born hippocampal granule neurons. Brain Behav Immun 2018;68:76-89.

9. Fourgeaud L, Través PG, Tufail Y, Leal-Bailey H, Lew ED, et al. TAM receptors regulate multiple features of microglial physiology. Nature 2016;532:240-4.

10. Sierra A, Encinas JM, Deudero JJ, Chancey JH, Enikolopov G, et al. Microglia shape adult hippocampal neurogenesis through apoptosis-coupled phagocytosis. Cell Stem Cell 2010;7:483-95.

11. Kettenmann H, Kirchhoff F, Verkhratsky A. Microglia: new roles for the synaptic stripper. Neuron 2013;77:10-8. 
12. Paolicelli RC, Bolasco G, Pagani F, Maggi L, Scianni M, et al. Synaptic pruning by microglia is necessary for normal brain development. Science 2011;333:1456-8.

13. Paolicelli RC, Gross CT. Microglia in development: linking brain wiring to brain environment. Neuron Glia Biol 2011;7:77-83.

14. Ginhoux F, Greter M, Leboeuf M, Nandi S, See P, et al. Fate mapping analysis reveals that adult microglia derive from primitive macrophages. Science 2010;330:841-5.

15. Hoeffel G, Ginhoux F. Ontogeny of tissue-resident macrophages. Front Immunol 2015;6:486.

16. Hanisch UK, Kettenmann H. Microglia: active sensor and versatile effector cells in the normal and pathologic brain. Nat Neurosci 2007;10:1387-94.

17. Herbomel P, Thisse B, Thisse C. Zebrafish early macrophages colonize cephalic mesenchyme and developing brain, retina, and epidermis through a M-CSF receptor-dependent invasive process. Dev Biol 2001;238:274-88.

18. Schlegelmilch T, Henke K, Peri F. Microglia in the developing brain: from immunity to behaviour. Curr Opin Neurobiol 2011;21:5-10.

19. Swinnen N, Smolders S, Avila A, Notelaers K, Paesen R, et al. Complex invasion pattern of the cerebral cortex bymicroglial cells during development of the mouse embryo. Glia 2013;61:150-63.

20. Buttgereit A, Lelios I, Yu X, Vrohlings M, Krakoski NR, et al. Sall1 is a transcriptional regulator defining microglia identity and function. Nat Immunol 2016;17:1397-406.

21. Kierdorf K, Erny D, Goldmann T, Sander V, Schulz C, et al. Microglia emerge from erythromyeloid precursors via Pu.1- and Irf8dependent pathways. Nat Neurosci 2013;16:273-80.

22. Askew K, Li K, Olmos-Alonso A, Garcia-Moreno F, Liang Y, et al. Coupled proliferation and apoptosis maintain the rapid turnover of microglia in the adult brain. Cell Rep 2017;18:391-405.

23. Réu P, Khosravi A, Bernard S, Mold JE, Salehpour M, et al. The lifespan and turnover of microglia in the human brain. Cell Rep 2017;20:779-84

24. Tay TL, Mai D, Dautzenberg J, Fernández-Klett F, Lin G, et al. A new fate mapping system reveals context-dependent random or clonal expansion of microglia. Nat Neurosci 2017;20:793-803.

25. Perry VH, Teeling J. Microglia and macrophages of the central nervous system: the contribution of microglia priming and systemic inflammation to chronic neurodegeneration. Semin Immunopathol 2013;35:601-12.

26. Thion MS, Ginhoux F, Garel S. Microglia and early brain development: an intimate journey. Science 2018;362:185-9.

27. VanRyzin JW, Pickett LA, McCarthy MM. Microglia: driving critical periods and sexual differentiation of the brain. Dev Neurobiol 2018;78:580-92.

28. Lenz KM, McCarthy MM. A starring role for microglia in brain sex differences. Neuroscientist 2015;21:306-21.

29. Hanamsagar R, Bilbo SD. Sex differences in neurodevelopmental and neurodegenerative disorders: focus on microglial function and neuroinflammation during development. J Steroid Biochem Mol Biol 2016;160:127-33.

30. Villa A, Gelosa P, Castiglioni L, Cimino M, Rizzi N, et al. Sex-specific features of microglia from adult mice. Cell Rep 2018;23:3501-11.

31. Welberg L. Microglia maketh the male. Nat Rev Neurosci 2013;14:226.

32. Garden GA, Campbell BM. Glial biomarkers in human central nervous system disease. Glia 2016;64:1755-71.

33. Lenz KM, Nugent BM, Haliyur R, McCarthy MM. Microglia are essential to masculinization of brain and behavior. J Neurosci 2013;33:2761-72.

34. Schafer DP, Lehrman EK, Stevens B. The "quad-partite" synapse: microglia-synapse interactions in the developing and mature CNS. Glia 2013;61:24-36

35. Forest MG, Cathiard AM, Bertrand JA. Total and unbound testosterone levels in the newborn and in normal and hypogonadal children: use of a sensitive radioimmunoassay for testosterone. J Clin Endocrinol Metab 1973;36:1132-42.

36. Reyes FI, Winter JS, Faiman C. Studies on human sexual development. I. Fetal gonadal and adrenal sex steroids. J Clin Endocrinol Metab 1973;37:74-8.

37. Wright CL, McCarthy MM. Prostaglandin E2-induced masculinization of brain and behavior requires protein kinase A, AMPA kainate, and metabotropic glutamate receptor signaling. J Neurosci 2009;29:13274-82.

38. McCarthy MM. Estradiol and the developing brain. Physiol Rev 2008;88:91-124.

39. Guneykaya D, Ivanov A, Hernandez DP, Haage V, Wojtas B, et al. Transcriptional and translational differences of microglia from male and female brains. Cell Rep 2018;24:2773-83.e6.

40. Bilbo SD. Sex differences in microglial appetites during development: Inferences and implications. Brain Behav Immun 2017;64:9-10.

41. Aanerud J, Borghammer P, Rodell A, Jónsdottir KY, Gjedde A. Sex differences of human cortical blood flow and energy metabolism. J Cereb Blood Flow Metab 2017;37:2433-40.

42. Esposito G, Van Horn JD, Weinberger DR, Berman KF. Gender differences in cerebral blood flow as a function of cognitive state with PET. J Nucl Med 1996;37:559-64.

43. Satterthwaite TD, Shinohara RT, Wolf DH, Hopson RD, Elliott MA, et al. Impact of puberty on the evolution of cerebral perfusion during adolescence. Proc Natl Acad Sci U S A 2014;111:8643-8.

44. Böttcher C, Schlickeiser S, Sneeboer MAM, Kunkel D, Knop A, et al. Human microglia regional heterogeneity and phenotypes determined by multiplexed single-cell mass cytometry. Nat Neurosci 2019;22:78-90.

45. Grabert K, Michoel T, Karavolos MH, Clohisey S, Baillie JK, et al. Microglial brain region-dependent diversity and selective regional sensitivities to aging. Nat Neurosci 2016;19:504-16.

46. Li Q, Cheng Z, Zhou L, Darmanis S, Neff NF, et al. Developmental heterogeneity of microglia and brain myeloid cells revealed by deep single-cell rna sequencing. Neuron 2019;101:207-23.e10.

47. Masuda T, Sankowski R, Staszewski O, Böttcher C, Amann L, et al. Spatial and temporal heterogeneity of mouse and human microglia at single-cell resolution. Nature 2019;566:388-92.

48. Lee SK. Sex as an important biological variable in biomedical research. BMB Rep 2018;51:167-73. 
49. GAO. Women's health-FDA needs to ensure more study of gender differences in prescription drug testing. 1992.

50. GBD 2016 Neurology Collaborators. Global, regional, and national burden of neurological disorders, 1990-2016: a systematic analysis for the Global Burden of Disease Study 2016. Lancet Neurol 2019;18:459-80.

51. Thibaut F. The role of sex and gender in neuropsychiatric disorders. Dialogues Clin Neurosci 2016;18:351-2.

52. Zagni E, Simoni L, Colombo D. Sex and gender differences in central nervous system-related disorders. Neurosci J 2016;2016:2827090.

53. Kopec AM, Smith CJ, Ayre NR, Sweat SC, Bilbo SD. Microglial dopamine receptor elimination defines sex-specific nucleus accumbens development and social behavior in adolescent rats. Nat Commun 2018;9:3769.

54. McCarthy MM, Pickett LA, VanRyzin JW, Kight KE. Surprising origins of sex differences in the brain. Horm Behav 2015;76:3-10.

55. Plassman BL, Langa KM, McCammon RJ, Fisher GG, Potter GG, et al. Incidence of dementia and cognitive impairment, not dementia in the United States. Ann Neurol 2011;70:418-26.

56. Seshadri S, Wolf PA, Beiser A, Au R, McNulty K, et al. Lifetime risk of dementia and Alzheimer's disease. The impact of mortality on risk estimates in the Framingham Study. Neurology 1997;49:1498-504.

57. Voskuhl RR, Gold SM. Sex-related factors in multiple sclerosis susceptibility and progression. Nat Rev Neurol 2012;8:255-63.

58. Nolen-Hoeksema S, Girgus JS. The emergence of gender differences in depression during adolescence. Psychol Bull 1994;115:424-43.

59. Altemus M, Sarvaiya N, Neill Epperson C. Sex differences in anxiety and depression clinical perspectives. Front Neuroendocrinol 2014;35:320-30.

60. Baldereschi M, Di Carlo A, Rocca WA, Vanni P, Maggi S, et al. Parkinson's disease and parkinsonism in a longitudinal study: two-fold higher incidence in men. ILSA Working Group. Italian Longitudinal Study on Aging. Neurology 2000;55:1358-63.

61. Elbaz A, Bower JH, Maraganore DM, McDonnell SK, Peterson BJ, et al. Risk tables for parkinsonism and Parkinson's disease. J Clin Epidemiol 2002;55:25-31.

62. del Aguila MA, Longstreth WT Jr, McGuire V, Koepsell TD, van Belle G. Prognosis in amyotrophic lateral sclerosis: a populationbased study. Neurology 2003;60:813-9.

63. McCombe PA, Henderson RD. Effects of gender in amyotrophic lateral sclerosis. Gend Med 2010;7:557-70.

64. Fombonne E. Epidemiological surveys of autism and other pervasive developmental disorders: an update. J Autism Dev Disord 2003;33:365-82.

65. Hattier MA, Matson JL, Tureck K, Horovitz M. The effects of gender and age on repetitive and/or restricted behaviors and interests in adults with autism spectrum disorders and intellectual disability. Res Dev Disabil 2011;32:2346-51.

66. Hiller RM, Young RL, Weber N. Sex differences in pre-diagnosis concerns for children later diagnosed with autism spectrum disorder. Autism 2016;20:75-84.

67. Bálint S, Czobor P, Komlósi S, Mészáros A, Simon V, et al. Attention deficit hyperactivity disorder (ADHD): gender- and age-related differences in neurocognition. Psychol Med 2009;39:1337-45.

68. Catalá-López F, Peiró S, Ridao M, Sanfélix-Gimeno G, Gènova-Maleras R, et al. Prevalence of attention deficit hyperactivity disorder among children and adolescents in Spain: a systematic review and meta-analysis of epidemiological studies. BMC Psychiatry 2012;12:168.

69. Cole WR, Mostofsky SH, Larson JC, Denckla MB, Mahone EM. Age-related changes in motor subtle signs among girls and boys with ADHD. Neurology 2008;71:1514-20.

70. Willcutt EG. The prevalence of DSM-IV attention-deficit/hyperactivity disorder: a meta-analytic review. Neurotherapeutics 2012;9:490-9.

71. Aleman A, Kahn RS, Selten JP. Sex differences in the risk of schizophrenia: evidence from meta-analysis. Arch Gen Psychiatry 2003;60:565-71.

72. Goldstein JM, Cherkerzian S, Tsuang MT, Petryshen TL. Sex differences in the genetic risk for schizophrenia: history of the evidence for sex-specific and sex-dependent effects. Am J Med Genet B Neuropsychiatr Genet 2013;162B:698-710.

73. Irvine K, Laws KR, Gale TM, Kondel TK. Greater cognitive deterioration in women than men with Alzheimer's disease: a meta analysis. J Clin Exp Neuropsychol 2012;34:989-98.

74. McGrath J, Saha S, Chant D, Welham J. Schizophrenia: a concise overview of incidence, prevalence, and mortality. Epidemiol Rev 2008;30:67-76.

75. Confavreux C, Vukusic S, Adeleine P. Early clinical predictors and progression of irreversible disability in multiple sclerosis: an amnesic process. Brain 2003;126:770-82.

76. Klein SL, Flanagan KL. Sex differences in immune responses. Nat Rev Immunol 2016;16:626-38.

77. Pinheiro I, Dejager L, Libert C. X-chromosome-located microRNAs in immunity: might they explain male/female differences? The X chromosome-genomic context may affect X-located miRNAs and downstream signaling, thereby contributing to the enhanced immune response of females. Bioessays 2011;33:791-802.

78. Fish EN. The X-files in immunity: sex-based differences predispose immune responses. Nat Rev Immunol 2008;8:737-44.

79. Wang X, Soloway PD, Clark AG. Paternally biased X inactivation in mouse neonatal brain. Genome Biol 2010;11:R79.

80. Carrel L, Willard HF. X-inactivation profile reveals extensive variability in X-linked gene expression in females. Nature 2005;434:400-4.

81. Souyris M, Cenac C, Azar P, Daviaud D, Canivet A, et al. TLR7 escapes X chromosome inactivation in immune cells. Sci Immunol $2018 ; 3$.

82. Lehmann SM, Krüger C, Park B, Derkow K, Rosenberger K, et al. An unconventional role for miRNA: let-7 activates Toll-like receptor 7 and causes neurodegeneration. Nat Neurosci 2012;15:827-35.

83. VanRyzin JW, Marquardt AE, Pickett LA, McCarthy MM. Microglia and sexual differentiation of the developing brain: a focus on extrinsic factors. Glia 2019.

84. Sierra A, Gottfried-Blackmore A, Milner TA, McEwen BS, Bulloch K. Steroid hormone receptor expression and function in microglia. Glia 2008;56:659-74. 
85. Benedusi V, Meda C, Della Torre S, Monteleone G, Vegeto E, et al. A lack of ovarian function increases neuroinflammation in aged mice. Endocrinology 2012;153:2777-88.

86. Sárvári M, Hrabovszky E, Kalló I, Solymosi N, Likó I, et al. Menopause leads to elevated expression of macrophage-associated genes in the aging frontal cortex: rat and human studies identify strikingly similar changes. J Neuroinflammation 2012;9:264.

87. Buss C, Entringer S, Swanson JM, Wadhwa PD. The role of stress in brain development: the gestational environment's long-term effects on the brain. Cerebrum 2012;2012:4.

88. Levesque ML, Fahim C, Ismaylova E, Verner MP, Casey KF. The impact of the in utero and early postnatal environments on grey and white matter volume: a study with adolescent monozygotic twins. Dev Neurosci 2015;37:489-96.

89. Yanguas-Casás N, Crespo-Castrillo A, de Ceballos ML, Chowen JA, Azcoitia I, et al. Sex differences in the phagocytic and migratory activity of microglia and their impairment by palmitic acid. Glia 2018;66:522-37.

90. Hanamsagar R, Bilbo SD. Environment matters: microglia function and dysfunction in a changing world. Curr Opin Neurobiol 2017;47:146-55.

91. Hanamsagar R, Alter MD, Block CS, Sullivan H, Bolton JL, et al. Generation of a microglial developmental index in mice and in humans reveals a sex difference in maturation and immune reactivity. Glia 2017; 65:1504-20.

92. Bordt EA, Ceasrine AM, Bilbo SD. Microglia and sexual differentiation of the developing brain: a focus on ontogeny and intrinsic factors. Glia 2019.

93. Nelson LH, Warden S, Lenz KM. Sex differences in microglial phagocytosis in the neonatal hippocampus. Brain Behav Immun 2017;64:11-22.

94. Wu LJ, Vadakkan KI, Zhuo M. ATP-induced chemotaxis of microglial processes requires P2Y receptor-activated initiation of outward potassium currents. Glia 2007;55:810-21.

95. McCarthy MM, Arnold AP. Reframing sexual differentiation of the brain. Nat Neurosci 2011;14:677-83.

96. Green PS, Simpkins JW. Neuroprotective effects of estrogens: potential mechanisms of action. Int J Dev Neurosci 2000;18:347-58.

97. Raghava N, Das BC, Ray SK. Neuroprotective effects of estrogen in CNS injuries: insights from animal models. Neurosci Neuroecon 2017;6:15-29.

98. Loram LC, Sholar PW, Taylor FR, Wiesler JL, Babb JA, et al. Sex and estradiol influence glial pro-inflammatory responses to lipopolysaccharide in rats. Psychoneuroendocrinology 2012;37:1688-99.

99. Smith AL, Alexander M, Rosenkrantz TS, Sadek ML, Fitch RH. Sex differences in behavioral outcome following neonatal hypoxia ischemia: insights from a clinical meta-analysis and a rodent model of induced hypoxic ischemic brain injury. Exp Neurol 2014;254:54-67.

100. Demarest TG, Schuh RA, Waddell J, McKenna MC, Fiskum G. Sex-dependent mitochondrial respiratory impairment and oxidative stress in a rat model of neonatal hypoxic-ischemic encephalopathy. J Neurochem 2016;137:714-29.

101. Mrdjen D, Pavlovic A, Hartmann FJ, Schreiner B, Utz SG, et al. High-dimensional single-cell mapping of central nervous system immune cells reveals distinct myeloid subsets in health, aging, and disease. Immunity 2018:48:599.

102. Hammond TR, Dufort C, Dissing-Olesen L, Giera S, Young A, et al. Single-cell RNA sequencing of microglia throughout the mouse lifespan and in the injured brain reveals complex cell-state changes. Immunity 2019;50:253-71.

103. Jordão MJC, Sankowski R, Brendecke SM, Sagar, Locatelli G, et al. Single-cell profiling identifies myeloid cell subsets with distinct fates during neuroinflammation. Science 2019;363:6425.

104. Keren-Shaul H, Spinrad A, Weiner A, Matcovitch-Natan O, Dvir-Szternfeld R, et al. A unique microglia type associated with restricting development of Alzheimer's disease. Cell 2017;169:1276-90.

105. Laskowitz DT, Thekdi AD, Thekdi SD, Han SK, Myers JK, et al. Downregulation of microglial activation by apolipoprotein E and apoE-mimetic peptides. Exp Neurol 2001;167:74-85.

106. Lynch JR, Tang W, Wang H, Vitek MP, Bennett ER, et al. APOE genotype and an ApoE-mimetic peptide modify the systemic and central nervous system inflammatory response. J Biol Chem 2003;278:48529-33.

107. Colton CA, Brown CM, Vitek MP. Sex steroids, APOE genotype and the innate immune system. Neurobiol Aging 2005;26:363-72.

108. Altmann A, Tian L, Henderson VW, Greicius MD; Alzheimer's Disease Neuroimaging Initiative Investigators. Sex modifies the APOE-related risk of developing Alzheimer disease. Ann Neurol 2014;75:563-73.

109. Cambronero FE, Liu D, Neal JE, Moore EE, Gifford KA, et al. APOE genotype modifies the association between central arterial stiffening and cognition in older adults. Neurobiol Aging 2018;67:120-7.

110. Parhizkar S, Arzberger T, Brendel M, Kleinberger G, Deussing M, et al. Loss of TREM2 function increases amyloid seeding but reduces plaque-associated ApoE. Nat Neurosci 2019;22:191-204.

111. Krasemann S, Madore C, Cialic R, Baufeld C, Calcagno N, et al. The TREM2-APOE pathway drives the transcriptional phenotype of dysfunctional microglia in neurodegenerative diseases. Immunity 2017;47:566-81.

112. Butovsky O, Jedrychowski MP, Cialic R, Krasemann S, Murugaiyan G, et al. Targeting miR-155 restores abnormal microglia and attenuates disease in SOD1 mice. Ann Neurol 2015;77:75-99.

113. Kang SS, Ebbert MTW, Baker KE, Cook C, Wang X, et al. Microglial translational profiling reveals a convergent APOE pathway from aging, amyloid, and tau. J Exp Med 2018;215:2235-45. 